\title{
Botulinum toxin injections as salvage therapy is beneficial for management of patellofemoral pain syndrome
}

Yuval Kesary ${ }^{1 *}$, Vivek Singh ${ }^{2}$ (0), Tal Frenkel-Rutenberg ${ }^{3}$, Arie Greenberg ${ }^{4}$, Shmuel Dekel ${ }^{1}$, Ran Schwarzkopf ${ }^{2}$ and Nimrod Snir ${ }^{1,5}$

\begin{abstract}
Purpose: Patellofemoral pain syndrome (PFPS) is a common pathology usually presenting with anterior or retropatellar pain. It is associated with a relative imbalance between the vastus medialis oblique (VMO) and the vastus lateralis $(\mathrm{VL})$ muscles. This can lead to considerable morbidity and reduced quality of life (QOL). This study aims to assess the long-term functional outcome of PFPS treated with VL muscle botulinum toxin A (BoNT-A) injection.

Materials and methods: A retrospective review was performed on 26 consecutive patients (31 knees) with a mean age of 50.1 years ( \pm 19.7 years) who were treated with BoNT-A injections to the VL muscle followed by physiotherapy between 2008 and 2015. Pre- and post-treatment pain levels (numerical rating scale, NRS), QOL (SF-6D), and functional scores (Kujala and Lysholm questionnaires) were measured. Demographics, physical therapy compliance, previous surgeries, perioperative complications, and patient satisfaction levels were collected.

Results: The mean follow-up time was $58.8 \pm 36.4$ months. There were significant improvements in all the examined domains. The average pain score (NRS) decreased from 7.6 to $3.2(P<0.01)$, and the Kujala, Lysholm, and SF-6D scores improved from 58.9 to $82.7(P<0.001)$, 56.2 to $83.2(P<0.001)$, and 0.6 to $0.8(P<0.001)$, respectively. Similar delta improvement was achieved irrespective of gender, age, compliance to post-treatment physical therapy, or coexisting osteoarthritis. Patients who presented with a worse pre-treatment clinical status achieved greater improvement. Prior to BoNT-A intervention, 16 patients (18 knees) were scheduled for surgery, of whom 12 (75\%, 13 knees) did not require further surgical intervention at the last follow-up.
\end{abstract}

Conclusions: A single intervention of BoNT-A injections to the VL muscle combined with physiotherapy is beneficial for the treatment of patients with persistent PFPS.

Level III evidence: Retrospective cohort study.

Keywords: Patella, Knee, Patellofemoral pain, Physical therapy, Botulinum toxin, Muscle imbalance

*Correspondence: ykesary@hotmail.com

1 Sackler Faculty of Medicine, Tel Aviv University, P. O. Box 39040, 6997801 Tel Aviv, Israel

Full list of author information is available at the end of the article

\section{Introduction}

Patellofemoral pain syndrome (PFPS) is a common condition that may cause severe retropatellar or peripatellar pain, making it the leading cause of anterior knee pain [1]. PFPS can be provoked during extensor mechanism activation during activities such as running, climbing stairs, squatting, and cycling. PFPS is reportedly more

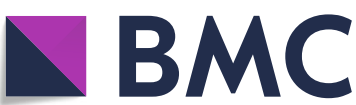

Part of Springer Nature

(c) The Author(s) 2021, corrected publication 2022. Open Access This article is licensed under a Creative Commons Attribution 4.0 International License, which permits use, sharing, adaptation, distribution and reproduction in any medium or format, as long as you give appropriate credit to the original author(s) and the source, provide a link to the Creative Commons licence, and indicate if changes were made. The images or other third party material in this article are included in the article's Creative Commons licence, unless indicated otherwise in a credit line to the material. If material is not included in the article's Creative Commons licence and your intended use is not permitted by statutory regulation or exceeds the permitted use, you will need to obtain permission directly from the copyright holder. To view a copy of this licence, visit http://creativecommons.org/licenses/by/4.0/. The Creative Commons Public Domain Dedication waiver (http://creativecommons.org/publicdomain/zero/1.0/) applies to the data made available in this article, unless otherwise stated in a credit line to the data. 
common in women, adolescents, and athletes, and it is one of the most common running-related injuries [2]. Although it accounts for $12 \%$ of the knee-related problems seen in general orthopedic practices [3] and up to $17 \%$ of the referrals to sports medicine specialty practices [2], information on PFPS prevalence and incidence in the general population is lacking [4].

The diagnosis of PFPS is based on patient history and physical examination, and it is determined by the presentation of peripatellar or retropatellar pain that is exacerbated with loading the patellofemoral joint (PFJ) while the knee is flexed. Additional PFPS symptoms can include crepitus, tenderness on patellar facet palpation, knee effusion, knee instability, buckling, and pain while sitting that is exacerbated when standing from a sitting position [4]. Correct diagnosis and treatment of PFPS may help preserve the affected individual's activity level, and decrease the prevalence of future patellofemoral degenerative changes [5]. PFPS has traditionally been considered a self-limiting condition, but current evidence now suggests that it may have a longer chronic course. Stathopulu et al. followed patients with PFPS during childhood and observed that $91 \%$ of them had chronic knee pain 22 years later [6].

PFPS is mainly treated non-surgically, with surgery usually being reserved for severe cases that failed to respond to non-surgical treatment [7]. While one Cochrane review found physiotherapy aimed at quadriceps strengthening to be an effective therapeutic approach [8], other modalities such as patellar taping [9], foot orthoses [10], and anti-inflammatory medications [7] were not found to be beneficial for long-term symptom relief. In contrast, other investigators have found that $40 \%$ of the patients treated by physiotherapy reported that their symptoms had persisted 1 year following treatment [11].

One of the principal mechanisms suggested for PFPS is a muscular imbalance between the vastus medialis oblique (VMO) and the vastus lateralis (VL) muscles. Previous studies have shown the benefit of botulinum type A (BoNT-A) injections not only on spastic disease $[12,14]$, but also to weaken selected muscle antagonist or agonist. BoNT-A is one of seven strains of the neurotoxin produced by Clostridium botulinum. Following intramuscular injection, BoNT-A produces a functional "denervation" via inhibition of acetylcholine release at the neuromuscular junction [15]. In animal models, muscle function recovery occurs over a 3 month period, due initially to formation of new terminal nerve sprouts and eventually to recovery of the parent terminal [15]. Therefore in theory, with the application of BoNT-A, the over-activated muscle tone of VL will be inhibited and the muscle imbalance between VMO and VL can then be altered, thus effectively correcting improper patellar tracking.

The objective of this study was to determine whether injecting botulinum type A (BoNT-A) to the VL muscle in combination with physiotherapy targeting the VMO muscle would alter the muscle balance and be an effective treatment for patients with anterior knee pain who had exhausted conservative treatment. We hypothesize that a single injection of BoNT-A to the VL muscle combined with physiotherapy will improve the patients with persistent PFPS.

\section{Materials and methods Study design}

A retrospective review was performed on 26 consecutive patients (31 knees) aged 22-84 (average age 49.9) years who were treated with BoNT-A injections to the VL muscle followed by physiotherapy at a single tertiary medical center between 2008 and 2015 and met the inclusion criteria. Data on patient demographics and the presence of coexisting osteoarthritis were recorded. The patients were diagnosed with PFPS based on history, physical examination, and assessment of advanced diagnostic imaging [17], all performed by the same experienced orthopedic surgeon. The physical examination included an active patella instability, which involved holding the knee in $15^{\circ}$ of flexion while the patient contracted the quadriceps muscle. A patellar lateral subluxation of more than $5 \mathrm{~mm}$ was regarded as a positive test result [19]. All patients had bilateral anteroposterior standing and lateral radiographs as well as a dynamic computerized tomographic (CT) scan consisting of four protocols: full extension with and without quadriceps contraction, and a $15^{\circ}$ flexed knee scan with and without quadriceps contraction [17].

BoNT-A injections were offered as an alternative to surgery to the patients who met all of the following inclusion criteria: (1) history of pain characteristic of PFPS, (2) radiological evidence of patellar subluxation, and (3) exhaustion of non-surgical treatment for more than a year. Radiologic assessment of patellar subluxation was made by measuring a congruence angle $>16^{\circ}$ (formed by a line bisecting the sulcus angle and a line drawn from the median ridge of the patella to the deepest point of the intercondylar sulcus) and $>2 \mathrm{~mm}$ distance between the margins of the medial pole of the patella and the medial trochlear facet or the median ridge of the patella with respect to the apex of the trochlear sulcus $[17,18]$. Patients who presented with concurrent knee pathology outside of the patellofemoral joint (PFJ) and those who did not meet the minimum of 6 months of follow-up were excluded from this analysis. The present study was 
conducted upon receiving approval from the institutional review board (IRB).

\section{Procedures}

The subjects were placed in a supine position. The patients received 500 units of BoNT-A (Dysport ${ }^{\circledR}$; Ipsen Biopharmaceuticals) divided into five injections that were administered into the distal part of the VL muscle at $3 \mathrm{~cm}$ intervals apart beginning $3-5 \mathrm{~cm}$ above the patella, on an oblique angle just lateral to the midline [19]. This was performed as an outpatient office procedure without image guidance (Fig. 1). Following the injections, the patients were started on a VMO muscle strengthening protocol utilizing open and closed kinematic chain exercises. A clinical follow-up evaluation was performed every 3 months.

\section{Outcome measures}

Patients were asked to complete functional and pain assessment questionnaires. The Kujala [20] and Lysholm Knee Scoring Scales [21] were used for functional assessment. The numerical rating scale (NRS) [22] and the SF-6D [26] questionnaires were used to evaluate pain and health-related quality of life. The primary outcome was defined as the delta change in the patient reported outcome measures (PROMs) prior to and following the intervention. The pre- and post-intervention analysis included comparisons of four subgroups (age, gender, presence of PFJ osteoarthritis, and compliance to postintervention physiotherapy). The effectiveness of intervention in each subgroup was assessed by measuring the

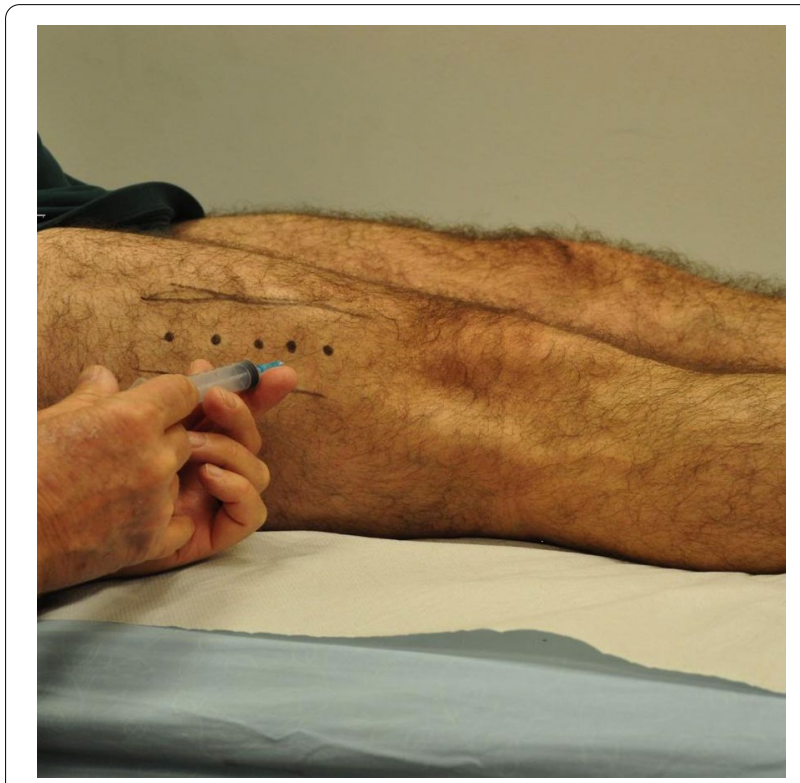

Fig. 1 BoNT-A injection site delta improvement in the scores of each questionnaire. The secondary outcome was avoidance of a pre-planned surgical intervention for PFPS based on the improvement achieved by the combined BoNT-A treatment and completed physiotherapy protocol.

\section{Statistical analysis}

All statistical analyses were performed using SPSS v24 (IBM Corporation, Armonk, New York). Paired-sample $t$-tests (normally distributed data) or related-sample Wilcoxon signed rank tests (skewed data) were used to compare changes between pre- and post-operation questionnaire scoring. Comparisons between sub-comparison groups (age, gender, coexisting osteoarthritis, and physiotherapy) were analyzed using independent sample $t$-tests (normally distributed data) or independent sample Mann-Whitney $U$ tests (skewed data). Pearson (normally distributed data) or Spearman correlations (skewed data) were used to analyze the correlations between preoperation questionnaire scores and changes in the questionnaire scores following the operation. Lastly, stepwise linear regression models were conducted to predict changes in questionnaire scores following the procedure.

\section{Results}

\section{Patient characteristics}

A total of 26 patients (31 knees) were included in the study, 10 females and 21 males. The average age was 50.1 years (range: $22-84$ years). The mean follow-up was 58.8 months (range: 11-104 months). Sixteen knees (52\%) were right-sided and 15 (48\%) were left-sided (Table 1). Coexisting osteoarthritis of the PFJ was confirmed by CT in 18 (58\%) knees prior to the initiation of treatment.

\section{Outcome measurements \\ Primary outcome}

The cohort showed statistically significant improvement in all patient reported outcomes (Table 2). The mean Lysholm score improved by $27.0 \pm 17.6$ points, the Kujala score improved by $23.8 \pm 17.0$ points, the

Table 1 Baseline characteristics of study participants

\begin{tabular}{ll}
\hline Characteristics & $\begin{array}{l}\text { Participants }(\boldsymbol{n}=\mathbf{2 6}, \\
\text { knees }=31)\end{array}$ \\
\hline Age $^{*}$, years & $50.1(22-84)$ \\
Follow up time*, months & $58.8(11-104)$ \\
Male: female, $n,(\%)$ & $10: 21(32 \%: 68 \%)$ \\
Injected leg (R: L), $n$ & $(16: 15)$ \\
Osteoarthritis: yes: no (\%) & $18: 13(58 \%: 42 \%)$ \\
\hline
\end{tabular}

*Values are expressed as mean (range) 
Table 2 Outcomes of the intervention

\begin{tabular}{llll}
\hline Questionnaire & $\begin{array}{l}\text { Pre }(\text { mean } \pm \text { SD) } \\
\text { Post (mean } \pm \text { SD) }\end{array}$ & $\boldsymbol{\Delta}($ mean \pm SD) & $\boldsymbol{P}$-value \\
\hline Lysholm & $56.2 \pm 15.0$ & $27.0 \pm 17.6$ & $<0.001$ \\
& $83.2 \pm 16.5$ & & \\
Kujala & $58.9 \pm 12.6$ & $23.8 \pm 17.0$ & $<0.001$ \\
& $82.7 \pm 15.2$ & & \\
NRS & $7.6 \pm 2.0$ & $-4.4 \pm 3.0$ & $<0.001$ \\
& $3.2 \pm 3.0$ & & \\
SF-6D & $0.6 \pm 0.1$ & $0.2 \pm 0.2$ & $<0.001$ \\
& $0.8 \pm 0.2$ & & \\
\hline
\end{tabular}

NRS numerical rating scale

SF-6D score improved by $0.2 \pm 0.2$ points, and the NRS score was reduced by $4.4 \pm 3.0$ points. $(P<0.001$ for all comparisons). These score differences are all clinically relevant as the established minimal clinically important difference (MCID) value pertaining to patellofemoral disorders for the Lysholm Knee Scoring Scale, Kujala, and SF-6D have been previously proposed as 10.1, 11.9, and 0.05 , respectively $24-26$.

Subgroup analysis When the patients were divided into two subgroups based on age ( $<60$ and $\geq 60$ years), there were statistically significant differences between all measured pre- and post-intervention scores evaluated $(P<0.05)$. Age was the sole variable to independently influence questionnaire score improvement in a linear regression model. Specifically, there was an inverse linear relation between patients' age and the Lysholm, Kujala, and SF-6D scores, but not the NRS score. The mean pre- and post-intervention Kujala and Lysholm scores in females were slightly lower compared with males. However, both males and females showed statistically significant differences between their preintervention compared with post-intervention scores for all of the measured variables $(P<0.05)$. Eighteen of the study participants had coexisting osteoarthritis compared with 13 who did not. Pre-intervention compared with post-intervention scores statistically differed for both these groups when considering all measured variables $(P<0.05)$.

Twenty-five of the 31 study knees $(81 \%)$ completed the physical therapy protocol aimed to strengthen the VMO muscle after the BoNT-A injections, and the remaining 6 (19\%) did not. Both subgroups showed statistically significant differences in Lysholm, Kujala, and NRS pre-intervention scores compared with their postintervention scores $(P<0.05)$. However, with regards to SF-6D scores, only the subgroup that completed the physiotherapy protocol had statistically significant differences between their pre- and post-intervention scores. All of these findings are summarized in Table 3.

Although not statistically significant, there were improvements in all questionnaire scores irrespective of age, gender, presence of osteoarthritis with PFJ, and compliance to physiotherapy among all subgroups that underwent BoNT-A treatment (Table 4). The scores of the younger subgroup ( $<60$ years) showed greater improvement compared with the older subgroup ( $\geq 60$ years) with regard to all measured variables. Female patients reported lower improvements in all measured scores compared with males. The presence or absence of osteoarthritis did not statistically affect the delta improvement for all questionnaire scores. Those who were compliant with the physiotherapy protocol showed a slightly higher improvement in Lysholm and Kujala scores compared with patients who were not, but these findings were not statistically different. Additionally, correlation analyses were performed to check the influence of the pre-intervention scores on measured improvement. The lower the Kujala, Lysholm, NRS, and SF-6D pre-intervention scores were, the greater the post-intervention improvement.

\section{Secondary outcome}

Sixteen patients (18 knees) were awaiting surgical intervention prior to the course of BoNT-A injections upon exhausting nonsurgical treatments over a period of 1 year. At the last follow-up, 12 patients (75\%, 13 knees) did not elect to undergo surgery owing to satisfactory pain relief and improved function. During all follow-up visits, no patient presented with complications or side effects related to the BoNT-A treatment.

\section{Discussion}

The results of the present study elucidate that BoNTA injections may be an effective and safe treatment for patients with persistent PFPS at midterm follow-up, regardless of the patient's age, gender, coexisting osteoarthritis, or compliance with physiotherapy following treatment. PFPS is a common knee pathology that negatively impacts an individual's QOL. An increased pressure on the PFJ, which can be related to a quadriceps muscle imbalance, likely causes it. Specifically, the etiology lies between the VMO muscle, which is the active medial stabilizer of the patella to the VL muscle, the iliotibial band, and the retinaculum, all of which apply forces in the lateral direction [27].

It has been suggested that the imbalance of the quadriceps muscle can be caused by a reduction of the VMO muscle force or by compromised temporal control of the VMO and VL muscle activity [28, 29]. Cowan et al. reported that PFPS patients demonstrated a delayed onset of electromyographic activity of the VMO muscle 
Table 3 Subgroup analysis - the intervention effectiveness in each group

\begin{tabular}{|c|c|c|c|c|c|c|c|c|}
\hline \multirow[t]{2}{*}{ Questionnaire } & \multicolumn{2}{|l|}{ Age group } & \multicolumn{2}{|l|}{ Gender } & \multicolumn{2}{|c|}{ Osteoarthritis } & \multicolumn{2}{|c|}{ Physiotherapy } \\
\hline & $\begin{array}{l}<60 \text { years } \\
(n=18)\end{array}$ & $\begin{array}{l}\geq 60 \text { years } \\
(n=13)\end{array}$ & $\begin{array}{l}\text { Male } \\
(n=10)\end{array}$ & $\begin{array}{l}\text { Female } \\
(n=21)\end{array}$ & $\begin{array}{l}\text { Yes } \\
(n=18)\end{array}$ & $\begin{array}{l}\text { No } \\
(n=13)\end{array}$ & $\begin{array}{l}\text { Yes } \\
(n=25)\end{array}$ & $\begin{array}{l}\text { No } \\
(n=6)\end{array}$ \\
\hline \multicolumn{9}{|l|}{ Lysholm } \\
\hline Pre & $56.4 \pm 16.9$ & $61.5 \pm 10.4$ & $58.7 \pm 17.6$ & $55.0 \pm 14.0$ & $57.9 \pm 12.9$ & $53.8 \pm 17.8$ & $54.9 \pm 14.5$ & $61.6 \pm 17.3$ \\
\hline Post & $82.6 \pm 18.8$ & $84.0 \pm 13.4$ & $89.8 \pm 11.5$ & $80.1 \pm 17.9$ & $80.7 \pm 19.1$ & $86.7 \pm 12.1$ & $82.5 \pm 17.0$ & $86.0 \pm 15.8$ \\
\hline$P^{* *}$ & $<0.001$ & 0.004 & 0.002 & $<0.001$ & $<0.001$ & $<0.001$ & $<0.001$ & 0.021 \\
\hline \multicolumn{9}{|l|}{ Kujala } \\
\hline Pre & $55.3 \pm 12.9$ & $63.9 \pm 10.9$ & $62.0 \pm 16.0$ & $57.5 \pm 10.8$ & $61.5 \pm 12.0$ & $55.3 \pm 13.1$ & $57.6 \pm 12.1$ & $64.6 \pm 14.5$ \\
\hline Post & $82.3 \pm 17.0$ & $83.2 \pm 13.0$ & $90.3 \pm 11.2$ & $79.1 \pm 15.8$ & $81.6 \pm 16.5$ & $84.2 \pm 13.7$ & $81.4 \pm 15.0$ & $88.2 \pm 16.5$ \\
\hline$P^{* *}$ & $<0.001$ & $<0.001$ & 0.002 & $<0.001$ & $<0.001$ & $<0.001$ & $<0.001$ & 0.032 \\
\hline \multicolumn{9}{|l|}{ NRS } \\
\hline Pre & $7.7 \pm 1.6$ & $7.4 \pm 2.5$ & $6.9 \pm 1.7$ & $7.9 \pm 2.1$ & $7.9 \pm 2.1$ & $7.2 \pm 1.9$ & $7.7 \pm 2.1$ & $7.2 \pm 1.5$ \\
\hline Post & $3.2 \pm 3.1$ & $3.2 \pm 2.9$ & $2.8 \pm 3.0$ & $3.4 \pm 3.0$ & $3.6 \pm 3.1$ & $2.7 \pm 2.8$ & $3.3 \pm 3.1$ & $3.0 \pm 2.8$ \\
\hline$P^{* *}$ & $<0.001$ & 0.001 & 0.002 & $<0.001$ & $<0.001$ & $<0.001$ & $<0.001$ & 0.042 \\
\hline \multicolumn{9}{|l|}{ SF-6 } \\
\hline Pre & $0.6 \pm 0.1$ & $0.7 \pm 0.1$ & $0.6 \pm 0.1$ & $0.6 \pm 0.1$ & $0.6 \pm 0.1$ & $0.6 \pm 0.1$ & $0.6 \pm 0.1$ & $0.7 \pm 0.2$ \\
\hline Post & $0.8 \pm 0.2$ & $0.8 \pm 0.1$ & $0.9 \pm 0.1$ & $0.7 \pm 0.2$ & $0.7 \pm 0.2$ & $0.8 \pm 0.1$ & $0.8 \pm 0.2$ & $0.8 \pm 0.1$ \\
\hline$P^{* *}$ & $<0.001$ & 0.019 & 0.02 & $<0.001$ & 0.005 & 0.001 & $<0.001$ & 0.171 \\
\hline
\end{tabular}

All data are presented as mean $\pm S D$

$P^{* *}$ - $p$-value of the comparison inside each subgroup. Refers to the Paired samples $t$-test (normal distribution) or related samples Wilcoxon signed rank test (skewed distribution)

Table 4 Subgroup analysis-improvement $\Delta$ comparison between the subgroups

\begin{tabular}{llllll}
\hline & Subgroups & Lysholm & Kujala & NRS & SF-6 \\
\hline $\begin{array}{llllll}\text { Age } \\
\text { group }\end{array}$ & $<60$ years & $30.5 \pm 19.0$ & $27.2 \pm 18.3$ & $-4.5 \pm 3.0$ & $0.2 \pm 0.2$ \\
& & & & & \\
& *60 years & $22.5 \pm 15.5$ & $18.9 \pm 14.4$ & $-4.2 \pm 3.2$ & $0.1 \pm 0.1$ \\
Gender & Male & 0.17 & 0.183 & 0.76 & 0.071 \\
& Female & $25.3 \pm 15.1$ & $21.4 \pm 14.6$ & $-4.5 \pm 3.1$ & $0.1 \pm 0.1$ \\
& $P^{*}$ & 0.574 & 0.268 & 0.753 & 0.297 \\
Osteoar- & Yes & $22.8 \pm 14.6$ & $19.8 \pm 14.5$ & $-4.3 \pm 3.0$ & $0.1 \pm 0.1$ \\
thritis & No & $33.2 \pm 20.6$ & $29.3 \pm 19.3$ & $-4.5 \pm 3.2$ & $0.2 \pm 0.1$ \\
& $P^{*}$ & 0.097 & 0.128 & 0.871 & 0.153 \\
Physio- & Yes & $27.8 \pm 18.1$ & $23.8 \pm 16.8$ & $-4.4 \pm 2.9$ & $0.1 \pm 0.1$ \\
therapy & No & $24.4 \pm 18.0$ & $23.5 \pm 19.5$ & $-4.2 \pm 3.8$ & $0.2 \pm 0.1$ \\
& $P^{*}$ & 0.827 & 0.975 & 0.869 & 0.854 \\
\hline
\end{tabular}

$\Delta$ Data represent the differences between the post- and pre-intervention scores. Values are expressed as mean \pm SD

$p^{*}$ the $p$-value of the subgroup comparison within each group. Refers to the independent sample $t$-test (normal distribution) or independent sample MannWhitney $U$ test (skewed distribution)

relative to the VL muscle [30]. In patients with a PFPS, the VL was activated earlier than the VMO when patients climbed downstairs and upstairs; however, in the control group that imbalance did not exist [30]. Although an objective parameter such as electromyographic activity was not evaluated in the present study, the clinical improvement experienced by patients may be adequate to justify BoNT-A as an effective option in treating PFPS. These findings have been supported by several other studies [29, 31, 32]. One previous study suggests that patients with patellofemoral problems exhibited atrophy of the VMO [33]. Furthermore, Van Tiggelen et al. in their prospective study determined that an activation delay of the VMO muscle was a risk factor to the development of PFPS in healthy soldiers [34]. Cloudon et al. demonstrated that the peak knee extension movement of PFPS patients was impaired compared with controls and that it reached normal values after directed treatment [35]. In addition, Besier et al. showed that the knee extension moments were reduced in PFPS patients during running compared with healthy controls [36]. Therefore, it is reasonable to assume that aiming to correct the VMO:VL.

One experimental way of changing the VMO:VL ratio is by injecting BoNT-A into the VL muscle combined with a tailored exercise program, thus preventing patellar maltracking through the achievement of normal muscle balance $[19,37-40]$. BoNT-A is a neurotoxin that weakens the muscle by blocking the pre-synaptic acetylcholine release, allowing weakness relative to the given dose of a muscle to be achieved for 12 weeks to 6 months [41-43]. 
The BoNT-A action is time-limited, and the muscle function is renewed by regeneration of new nerve sprouts within one month [44]. Previous studies have established the utility of intramuscular injection of BoNT-A to address focal muscle overactivity to manage neurological conditions [45]; however, its use for treatment of musculoskeletal conditions is not as well reported in the literature [38]. In the setting of PFPS, BoNT-A injections have the potential to address the proposed imbalance between a relatively overactive VL muscle and its less active synergists, including the VMO muscle. This provides a unique opportunity for aimed muscle re-training and rebuilding of more normal quadriceps muscle activation patterns and joint function. We believe that this is more viably accomplished with explicit and centered muscle re-training fortified by expert healthcare providers, rather than just from ordinary every day movements.

To date, data on BoNT-A treatment for PFPS is relatively scarce [19, 37, 39, 40, 42, 43]. We designed this study to expand current knowledge by exploring treatment outcomes in affected patients. Singer et al. compared 14 patients treated with electromyography-guided BoNT-A injections with 10 patients who received a placebo, both followed by a physiotherapy protocol [46]. The authors reported that the treatment was beneficial in terms of improved pain and function [46]. A followup study of the same group showed that patient-reported improvement lasted for an average of 34 months [40]. However, it should be mentioned that both of those studies were industry-funded by Ipsen Biopharmaceuticals, the manufacturer of Dysport. Chen et al. reported on 12 patients with bilateral PFPS that showed improvement in pain, stiffness, and function of the knee after being injected with BoNT-A compared with the contralateral non-injected side at 3 months follow-up [39]. Additionally, Stephen et al. aimed to investigate the effect of an ultrasound-guided BoNT-A injection into the tensor fasciae latae (TFL) in patients with lateral patellofemoral overload syndrome (LPOS) who failed conventional treatment [47]. They concluded that an injection of BoNT-A into the TFL, combined with physical therapy resulted in a significant improvement of symptoms in patients with LPOS, which was maintained at 5-year follow-up [47].

The above-cited studies describe the use of various guiding methods to determine the injection site, which may make these treatment options more expensive and less suitable for an office procedure [37]. In our study, we used anatomic landmarks, making it more suitable for an office procedure and potentially increasing its availability. Unlike previous studies that excluded older patients ( $>60$ years) and patients with PFJ osteoarthritis [46], we included both of these more challenging patient populations and found that they too benefited from the BoNT-A injection and physical therapy protocol. However, the treatment was most successful in the younger age group and in patients who presented with a worse clinical status. Notably, PFPS is more common in the younger, more active population. This supports the rationale of using BoNT-A injections as a salvage therapy for patients who had exhausted the available nonsurgical treatments and who planned to undergo surgery. This was further validated in the present study as many patients in our cohort canceled their planned surgical interventions as a result of successful BoNT-A treatment. Potential adverse effect of the treatment was hypothesized to be an initial reduction in quadriceps femoris muscle strength due to the reduced contribution of the distal VL muscle. This was of concern since it has been demonstrated that significant quadriceps femoris muscle weakness may already exist in the affected limb of individuals with PFJ dysfunction [48].

There are several limitations to this study. First, due to its retrospective nature, the study was exposed to memory bias of the participants when responding to the questionnaires. The subgroup analyses may be subject to low statistical power due to the limited number of participants. Body mass index and other pre-existing comorbidities in the form of American Society of Anesthesiologists' (ASA) classification and Charlson Comorbidity Index (CCI) were not evaluated. Additionally, a matched non-treatment cohort was not available, which would have allowed for a more in-depth analysis of the effectiveness of BoNT-A injections. Fluoroscopic or ultrasound guided injection was not utilized in the study to improve potential accuracy. A greater number of females were examined compared with male patients; PFPS is thought to more commonly affect female patients, so this may be representative [49]. Lastly, this study was performed in a single medical clinic, thus limiting its external validity. Despite these limitations, the findings of our study are promising and can be used as a platform for future investigations on this topic.

\section{Conclusions}

Treatment of PFPS by utilizing BoNT-A injections provides a cost and time effective alternative to not only ongoing conservative management but also surgical intervention for patients experiencing refractory anterior knee pain. It significantly improved functional outcomes and reduced pain in patients who had exhausted conservative treatment methodologies. However, larger prospective studies are needed to evaluate the effectiveness of the herein described intervention and characterize the patients who will benefit most from it. 


\section{Abbreviations}

PFPS: Patellofemoral pain syndrome; VMO: Vastus medialis oblique muscle; VL: Vastus lateralis muscle; QOL: Quality of life; BoNT-A: Botulinum toxin A; PFJ: Patellofemoral joint; NRS: Numerical rating scale.

\section{Funding}

No funding was provided for this study.

\section{Availability of data and materials}

The datasets generated during and/or analyzed during the current study are available from the corresponding author on reasonable request.

\section{Declarations}

\section{Ethical approval and consent to participate}

The present study was conducted upon receiving approval from the institutional review board (IRB). Informed consent was obtained from all individual participants included in the study.

\section{Consent for publication}

Consent to publish was obtained from all individual participants included in the study.

\section{Competing interests}

Y.K., V.S., T.F.R., A.G., S.D., and N.S. have nothing to disclose. R.S. is a paid consultant for Intellijoint and Smith \& Nephew and holds stock options in Gauss Surgical.

\section{Author details}

${ }^{1}$ Sackler Faculty of Medicine, Tel Aviv University, P. O. Box 39040, 6997801 Tel Aviv, Israel. ${ }^{2}$ Department of Orthopedic Surgery, NYU Langone Health, NYU Langone Orthopedic Hospital, New York, NY, USA. ${ }^{3}$ Orthopedic Department, Rabin Medical Center, Beilinson Hospital, Petah Tikva, Israel. ${ }^{4}$ Department of Orthopedic Surgery, Kaplan Medical Center, Rehovot, Israel. ${ }^{5}$ Division of Adult Reconstruction, Department of Orthopedics, Tel Aviv Sourasky Medical Center, Tel Aviv, Israel.

Received: 13 July 2021 Accepted: 14 October 2021 Published online: 29 October 2021

\section{References}

1. Petersen W, Ellermann A, Gösele-Koppenburg A, Best R, Rembitzki IV, Brüggemann GP et al (2014) Patellofemoral pain syndrome. Knee Surg Sports Traumatol Arthrosc. https://doi.org/10.1007/s00167-013-2759-6

2. Taunton JE (2002) A retrospective case-control analysis of 2002 running injuries. Br J Sports Med 36(2):95-101

3. Wood L, Muller S, Peat G (2011) The epidemiology of patellofemoral disorders in adulthood: a review of routine general practice morbidity recording. Prim Health Care Res Dev 12(2):157-164

4. Crossley KM, Stefanik JJ, Selfe J, Collins NJ, Davis IS, Powers CM et al (2016) 2016 Patellofemoral pain consensus statement from the 4th international patellofemoral pain research retreat, Manchester. Part 1: Term 1. Br J Sports Med 50(14):839-843

5. Thomas MJ, Wood L, Selfe J, Peat G (2010) Anterior knee pain in younger adults as a precursor to subsequent patellofemoral osteoarthritis: a systematic review. BMC Musculoskelet Disord 11:201

6. Stathopulu E, Baildam E (2003) Anterior knee pain: a long-term followup. Rheumatology (Oxford) 42(2):380-382

7. Heintjes E, Berger MY, Bierma-Zeinstra SM, Bernsen RMD, Verhaar JN, Koes BW (2004) Pharmacotherapy for patellofemoral pain syndrome. Cochrane database of systematic reviews (Online). (3):CD003470.

8. van der Heijden RA, Lankhorst NE, van Linschoten R, Bierma-Zeinstra SMA, van Middelkoop M (2015) Exercise for treating patellofemoral pain syndrome. Cochrane Database Syst Rev. https://doi.org/10.1002/ 14651858.CD010387.pub2

9. Callaghan MJ, Selfe J (2012) Patellar taping for patellofemoral pain syndrome in adults. Cochrane Database Syst Rev. https://doi.org/10.1002/ 14651858.CD006717.pub2
10. Hossain M, Alexander P, Burls A, Jobanputra P (2011) Foot orthoses for patellofemoral pain in adults. Cochrane Database Syst Rev. https://doi. org/10.1002/14651858.CD008402.pub2

11. Witvrouw E, Callaghan MJ, Stefanik JJ, Noehren B, Bazett-Jones DM, Willson JD et al (2014) Patellofemoral pain: consensus statement from the 3rd international patellofemoral pain research retreat held in Vancouver, September 2013. Br J Sports Med 48(6):411-414

12. Paparella G, Vavla M, Bernardi L, Girardi G, Stefan C, Martinuzzi A (2020) Efficacy of a combined treatment of botulinum toxin and intensive physiotherapy in hereditary spastic paraplegia. Front Neurosci. https://www. frontiersin.org/article/https://doi.org/10.3389/fnins.2020.00111/full

13. Rousseaux M, Launay MJ, Kozlowski O, Daveluy W (2007) Botulinum toxin injection in patients with hereditary spastic paraparesis. Eur J Neurol. https://onlinelibrary.wiley.com/doi/https://doi.org/10.1111/j.1468-1331. 2006.01617.x

14. Simpson LL. The origin, structure, and pharmacological activity of botulinum toxin. http://www.ncbi.nlm.nih.gov/pubmed/6119708

15. Rogozhin AA, Pang KK, Bukharaeva E, Young C, Slater CR (2008) Recovery of mouse neuromuscular junctions from single and repeated injections of botulinum neurotoxin A. J Physiol. http://doi.wiley.com/https://doi. org/10.1113/jphysiol.2008.153569

16. Gulati A, McElrath C, Wadhwa V, Shah JP, Chhabra A (2018) Current clinical, radiological and treatment perspectives of patellofemoral pain syndrome. Br J Radiol. http://www.ncbi.nlm.nih.gov/pubmed/29303366

17. Manske RC, Davies GJ. Examination of the patellofemoral joint. http:// www.ncbi.nlm.nih.gov/pubmed/27904788

18. Endo Y, Stein BES, Potter HG (2011) Radiologic assessment of patellofemoral pain in the athlete. Sports Health. http://journals.sagepub.com/ doi/https://doi.org/10.1177/1941738110397875

19. Drake DF, Pidcoe PE, Ericksen J (2011) Botulinum toxin type A for nonsurgical lateral release in patellofemoral pain syndrome: a case study. Military Med. https://academic.oup.com/milmed/article/176/6/696-698/ 4345435

20. Kujala UM, Jaakkola LH, Koskinen SK, Taimela S, Hurme M, Nelimarkka O (1993) Scoring of patellofemoral disorders. Arthroscopy 9(2):159-163

21. Collins NJ, Misra D, Felson DT, Crossley KM, Roos EM (2011) Measures of knee function: International Knee Documentation Committee (IKDC) Subjective Knee Evaluation Form, Knee Injury and Osteoarthritis Outcome Score (KOOS), Knee Injury and Osteoarthritis Outcome Score Physical Function Short Form (KOOS-PS). Knee Ou Arthritis Care Res 63(Suppl 1):S208-S228

22. Hjermstad MJ, Fayers PM, Haugen DF, Caraceni A, Hanks GW, Loge JH, et al (2011) Studies comparing numerical rating scales, verbal rating scales, and visual analogue scales for assessment of pain intensity in adults: a systematic literature review. J Pain Symptom Manag. https://linki nghub.elsevier.com/retrieve/pii/S0885392411000145

23. Brazier JE, Roberts J. The estimation of a preference-based measure of health from the SF-12. https://journals.IwW.com/00005650-20040 9000-00004

24. Irrgang J et al (2012) Summary of clinical outcome measures for sportsrelated knee injuries: final report. AOSSM Outcomes Task Force. https:// www.sportsmed.org/AOSSMIMIS/members/downloads/research/Clini calOutcomeMeasuresKnee pdf.

25. Jones KJ, Kelley B V, Arshi A, McAllister DR, Fabricant PD (2019) Comparative effectiveness of cartilage repair with respect to the minimal clinically important difference. http://www.ncbi.n/m.nih.gov/pubmed/31082325

26. Walters SJ, Brazier JE (2003) What is the relationship between the minimally important difference and health state utility values? The case of the SF-6D. http://www.ncbi.nlm.nih.gov/pubmed/12737635

27. Collado H, Fredericson M (2010) Patellofemoral pain syndrome. Clin Sports Med 29(3):379-398

28. Hug F, Hodges PW, Tucker K (2015) Muscle force cannot be directly inferred from muscle activation: illustrated by the proposed imbalance of force between the vastus medialis and vastus lateralis in people with patellofemoral pain. J Orthop Sports Phys Ther. http://www.jospt.org/ doi/https://doi.org/10.2519/jospt.2015.5905

29. Chen H-Y, Chien C-C, Wu S-K, Liau J-J, Jan M-H (2012) Electromechanical delay of the vastus medialis obliquus and vastus lateralis in individuals with patellofemoral pain syndrome. J Orthop Sports Phys Ther. http:// www.jospt.org/doi/https://doi.org/10.2519/jospt.2012.3973 
30. Cowan SM, Bennell KL, Hodges PW, Crossley KM, McConnell J (2001) Delayed onset of electromyographic activity of vastus medialis obliquus relative to vastus lateralis in subjects with patellofemoral pain syndrome. Arch Phys Med Rehabil 82(2):183-189

31. Cavazzuti L, Merlo A, Orlandi F, Campanini I (2010) Delayed onset of electromyographic activity of vastus medialis obliquus relative to vastus lateralis in subjects with patellofemoral pain syndrome. Gait Posture. https://linkinghub.elsevier.com/retrieve/pii/S0966636210002109

32. Witvrouw E, Lysens R, Bellemans J, Cambier D, Vanderstraeten G (2000) Intrinsic risk factors for the development of anterior knee pain in an athletic population: a two-year prospective study. Am J Sports Med. http:// journals.sagepub.com/doi/https://doi.org/10.1177/036354650002800 40701

33. Pattyn E, Verdonk P, Steyaert A, Vanden Bossche L, Van den Broecke W, Thijs Y, et al (2011) Vastus medialis obliquus atrophy: does it exist in patellofemoral pain syndrome? Am J Sports Med. http://journals.sagepub. com/doi/https://doi.org/10.1177/0363546511401183

34. Van Tiggelen D, Cowan S, Coorevits P, Duvigneaud N, Witvrouw E (2009) Delayed vastus medialis obliquus to vastus lateralis onset timing contributes to the development of patellofemoral pain in previously healthy men: a prospective study. Am J Sports Med 37(6):1099-1105

35. Claudon B, Poussel M, Billon-Grumillier C, Beyaert C, Paysant J (2012) Knee kinetic pattern during gait and anterior knee pain before and after rehabilitation in patients with patellofemoral pain syndrome. Gait Posture 36(1):139-143

36. Besier TF, Fredericson M, Gold GE, Beaupre GS, Delp SL (2009) Knee muscle forces during walking and running in patellofemoral pain patients and pain-free controls. J Biomech 42(7):898-905

37. Singer B, Silbert B, Silbert P, Singer K (2015) The role of botulinum toxin type $\mathrm{a}$ in the clinical management of refractory anterior knee pain. Toxins. http://www.mdpi.com/2072-6651/7/9/3388

38. Cullen DM, Boyle JJW, Silbert PL, Singer BJ, Singer KP (2007) Botulinum toxin injection to facilitate rehabilitation of muscle imbalance syndromes in sports medicine. http://www.tandfonline.com/doi/full/https://doi.org/ 10.1080/09638280701568627

39. Chen JTN, Tang ACW, Lin SC, Tang SFT (2015) Anterior knee pain caused by patellofemoral pain syndrome can be relieved by Botulinum toxin type A injection. Clin Neurol Neurosurg 129:S27-29

40. Silbert BI, Singer BJ, Silbert PL, Gibbons JT, Singer KP (2012) Enduring efficacy of Botulinum toxin type A injection for refractory anterior knee pain. Disabil Rehabil 34(1):62-68

41. Sim WS (2011) Application of botulinum toxin in pain management. Korean J pain. http://www.ncbi.nlm.nih.gov/pubmed/21390172

42. Singer BJ, Silbert PL, Dunne JW, Song S, Singer KP (2006) An open label pilot investigation of the efficacy of Botulinum toxin type A [Dysport] injection in the rehabilitation of chronic anterior knee pain. http://www. tandfonline.com/doi/full/https://doi.org/10.1080/09638280500301477

43. Singer BJ, Silbert PL, Song S, Dunne JW, Singer KP (2011) Treatment of refractory anterior knee pain using botulinum toxin type $A$ (Dysport) injection to the distal vastus lateralis muscle: a randomised placebo controlled crossover trial. https://bjsm.bmj.com/lookup/doi/https://doi. org/10.1136/bjsm.2009.069781

44. de Paiva A, Meunier FA, Molgo J, Aoki KR, Dolly JO (1999) Functional repair of motor endplates after botulinum neurotoxin type A poisoning: biphasic switch of synaptic activity between nerve sprouts and their parent terminals. Proc Natl Acad Sci USA 96(6):3200-3205

45. Simpson DM, Gracies J-M, Graham HK, Miyasaki JM, Naumann M, Russman B, et al (2008) Assessment: Botulinum neurotoxin for the treatment of spasticity (an evidence-based review): [RETIRED]. http://www.neurology.org/lookup/doi/https://doi.org/10.1212/01.wnl.0000311391.00944.c4

46. Singer BJ, Silbert PL, Song S, Dunne JW, Singer KP (2011) Treatment of refractory anterior knee pain using botulinum toxin type A (Dysport) injection to the distal vastus lateralis muscle: a randomised placebo controlled crossover trial. Br J Sports Med 45(8):640-645

47. Stephen JM, Urquhart DWJ, van Arkel RJ, Ball S, Jaggard MKJ, Lee JC, et al (2016) The use of sonographically guided botulinum toxin type A (Dysport) injections into the tensor fasciae latae for the treatment of lateral patellofemoral overload syndrome. Am J Sports Med. http://journals. sagepub.com/doi/https://doi.org/10.1177/0363546516629432

48. Giles LS, Webster KE, McClelland JA, Cook J (2013) Does quadriceps atrophy exist in individuals with patellofemoral pain? A systematic literature review with meta-analysis. J Orthopaed Sports Phys Therapy. http://www. jospt.org/doi/https://doi.org/10.2519/jospt.2013.4833

49. Boling M, Padua D, Marshall S, Guskiewicz K, Pyne S, Beutler A (2010) Gender differences in the incidence and prevalence of patellofemoral pain syndrome. http://doi.wiley.com/https://doi.org/10.1111/j.1600-0838. 2009.00996.x

\section{Publisher's Note}

Springer Nature remains neutral with regard to jurisdictional claims in published maps and institutional affiliations.
Ready to submit your research? Choose BMC and benefit from:

- fast, convenient online submission

- thorough peer review by experienced researchers in your field

- rapid publication on acceptance

- support for research data, including large and complex data types

- gold Open Access which fosters wider collaboration and increased citations

- maximum visibility for your research: over 100M website views per year

At BMC, research is always in progress.

Learn more biomedcentral.com/submissions 